OPEN ACCESS

Edited by:

Bin Zhang,

Southern Medical University, China

Reviewed by:

Lian Du,

First Affiliated Hospital of Chongqing Medical University, China

Sha Liu,

First Hospital of Shanxi Medical

University, China

*Correspondence:

Qiang Wang

wangqiang130@scu.edu.cn

tThese authors have contributed equally to this work and share first authorship

Specialty section:

This article was submitted to

Sleep Disorders,

a section of the journal

Frontiers in Psychiatry

Received: 25 September 2021

Accepted: 28 October 2021

Published: 02 December 2021

Citation:

Xie M, Tang Y, Zhu L, Dai M, Wu Y,

Huang Y, Liu Y, Xiao L, Li T and

Wang Q (2021) Childhood Trauma

and Mental Health Status in General

Population: A Series Mediation

Examination of Psychological Distress

in COVID-19 Pandemic and Global

Sleep Quality

Front. Psychiatry 12:782913.

doi: 10.3389/fpsyt.2021.782913

\section{Childhood Trauma and Mental Health Status in General Population: A Series Mediation Examination of Psychological Distress in COVID-19 Pandemic and Global Sleep Quality}

\author{
Min Xie ${ }^{1+}$, Yiguo Tang ${ }^{1+}$, Ling $\mathrm{Zhu}^{2+}$, Minhan Dai ${ }^{1}$, Yulu $\mathrm{Wu}^{1}$, Yunqi Huang ${ }^{1}$, Yunjia Liu ${ }^{1}$, \\ Liling Xiao ${ }^{1}$, Tao $\mathrm{Li}^{1,3}$ and Qiang Wang ${ }^{1 *}$
}

${ }^{1}$ Mental Health Centre and Psychiatric Laboratory, West China Hospital, Sichuan University, Chengdu, China, ${ }^{2}$ Department of Clinical Psychology, Southwest Hospital, The First Hospital Affiliated to Army Medical University, Third Military Medical University, Chongaing, China, ${ }^{3}$ Affiliated Mental Health Centre and Hangzhou Seventh People's Hospital, Zhejiang University School of Medicine, Hangzhou, China

Background: Coronavirus-2019 (COVID-19) has been coexisting with humans for almost 2 years, consistently impacting people's daily life, medical environment, and mental health. This study aimed to test the series mediation model triggered by childhood trauma, in which perceived psychological impact of COVID-19 pandemic and sleep quality mediated the path sequentially and led to adverse mental health outcomes.

Methods: A cross-sectional design involving 817 participants were enrolled via WeChat online survey. Participants completed questionnaires, including demographic features, the Childhood Trauma Questionnaire, Impact of Event Scale-Revised (IES-R) questionnaire, Pittsburgh Sleep Quality Index (PSQI) questionnaire, and Depression, Anxiety, and Stress Scale (DASS-21). Pearson correlations and hierarchical multiple linear regression were employed to examine the association of childhood trauma and psychological stress of COVID-19, sleep quality, and mental health status. In addition, a series mediate analysis was carried out to examine sequence mediating effects of psychological impact of COVID-19 and sleep quality between childhood trauma and mental health status.

Results: The results showed that childhood trauma is positively and significantly related to psychological distress of COVID-19 pandemic, sleep quality, and mental health status $(p<0.05)$. Hierarchical multiple linear regression analysis shown that demographic features explained 4.4, 2.1, and $4.0 \%$ of the total variance in DASS-21, IES-R, and PSQI total scale scores, respectively. Adding childhood trauma significantly increased the model variance of DASS-21 $\left(\Delta R^{2}=0.129, F\right.$ $=126.092, p=0.000)$, IES-R $\left(\Delta R^{2}=0.062, F=54.771, p=0.000\right)$, and PSQl total scale scores $\left(\Delta R^{2}=0.055, F=48.733, p=0.000\right)$, respectively. Moreover, the series mediation model showed that the perceived impact of the COVID-19 pandemic and sleep quality were sequential mediators between childhood trauma and mental health status (proportion explained: 49.17\%, $p<0.05$ ). 


\begin{abstract}
Conclusion: Amid the ravages of COVID-19, childhood trauma predicts poor mental health status, in part because of greater psychological impact related to COVID-19 and poorer global sleep quality. In order to improve mental health, future researchers should pay more attention to individuals with childhood trauma, for its association with greater stress related to life events and poorer sleep quality.
\end{abstract}

Keywords: coronavirus disease 2019, sleep quality, childhood trauma, depression, anxiety

\section{INTRODUCTION}

Globally, 2019 coronavirus disease (COVID-19), a highly infectious and potentially fatal disease, has been coexisting with humans for almost 2 years. Previous studies found that COVID-19 has a sudden and massive impact on freedom of movement, daily activity, and medical environment, which could significantly ruin mental health of medical staff, patients with mental disorder, and the general population $(1-5)$. What is more, one meta-analysis found that the overall prevalence of sleep disturbances, depression, and anxiety among COVID-19 patients is 34,45 , and $47 \%$, respectively (6). One study across geographic regions worldwide reported that events related to COVID-19 were more likely to be associated with mental health symptoms, especially symptoms of post-traumatic stress disorder (PTSD), insomnia, depression, and anxiety in the general population (7).

Childhood trauma, emotional or physical adverse experiences in one's early life, was associated with increased risk for developing almost all mental disorders, including sleep disorders, depression, anxiety, bipolar disorder, PTSD, and schizophrenia $(8-10)$. The extant literature suggests that stress exposure during early life may lead to excessive glucocorticoid release, dysfunction of hypothalamic-pituitary-adrenal (HPA) axis, abnormal development of brain trajectories, and changes of epigenetics regulation (11-14). The trauma-psychosis cycle proposed that individual exposures to environmental stressors during early life further impaired their adaptive coping strategies and thus increased the vulnerability to future stressors (15). Stress-sensitization model proposed that dysregulation of stress response caused by exposure to childhood trauma may render an individual more susceptible to psychosis triggered by later stressors (16).

Some previous studies have reported that historical trauma (physical and emotional trauma over the life span and across generations), childhood abuse, and social support were closely related to psychological stress, sleep quality, and emotion regulation during the COVID-19 outbreak (17-19). One study found that psychological stress of COVID-19 mediated the association between childhood trauma and Pittsburgh Sleep Quality Index (PSQI) global sleep quality (18). Another study found that the relationship between childhood trauma and the severity of depressive/anxiety symptoms was partly mediated by insomnia symptoms in severe mental disorders (20). However, our understanding of how traumatic stress symptoms related to COVID-19 and global sleep quality mediate the association between early life trauma and mental health symptoms is currently limited. So, we aim to (1) examine the association between childhood trauma and psychological impact related to COVID-19, sleep quality, and the mental health condition in the general population and (2) explore whether there are mediating effects of traumatic symptoms related to COVID-19 and global sleep quality between childhood trauma and mental health status.

\section{METHODS}

\section{Design and Participants}

A cross-sectional survey-based study was conducted from August 20, 2021 to September 5, 2021. All participants in this study were recruited via WeChat, the most widely used social media platform in China, and all data were collected using electronic questionnaires via online survey tool, Wenjuanxing platform (https://www.wjx.cn/app/survey.aspx). In order to obtain more participation from different regions of China, direct online and snowball recruitment methods through "Circle of Friends" of WeChat were used. There were 948 participants who voluntarily filled in and submitted the questionnaire, among which $52.89,9.15,8.83,5.68,4.31,2.84,2.63$, and $2.52 \%$ were from Sichuan, Chongqing, Henan, Hebei, Liaoning, Beijing, Jiangsu, and Guangdong, respectively. Our inclusion criteria were as follows: (1) aged from 16 to 60 years old and (2) were able to use smart phones and complete questionnaires. Invalid and incomplete questionnaires were excluded. The childhood trauma questionnaire has seven reverse-scored items, five of which constitute the subscale of emotional neglect (EN). Based on the comparison between the scores of reverse-scored items and the forward-scored items, we can better exclude invalid questionnaires (21). The questionnaires included general demographic characteristics (age, gender, BMI, education level, income level), childhood trauma, mental health (sleep quality, depressive symptoms, and COVID-19 related traumatic stress symptoms), and mental disorders (anxiety, depression, bipolar disorder, and schizophrenia) diagnosed by psychiatrists.

\section{Measures}

The education was divided into nine levels, including illiteracy (1), primary school (2), middle school (3), vocational high school (4), senior high school (5), junior college (6), bachelor's degree (7), master's degree (8), and doctor's degree/Ph.D. (9). The income levels were collected as self-reported incomes compared with those of local people. It was divided into five categories: $1=$ Low income level; $2=$ Low-Middle income level; $3=$ Middle income level; $4=$ Middle-High income level; and $5=$ High income level. Education and income level were covariates 
in the subsequent hierarchical linear regression analysis and mediation analysis.

\section{Childhood Trauma Questionnaires}

Childhood trauma questionnaires-short form (CTQ-SF) was adopted to assess participants' experience from emotional abuse (EA), physical abuse (PA), sexual abuse (SA), EN, and physical neglect (PN) before 16 years old (21). A five-point Likert scale ( $1=$ not at all, $5=$ very often $)$ was used to indicate the trauma severity about certain events or situations occurring during the childhood. Childhood trauma questionnaires-short form consisted of 25 clinical items and 3 validity items. The 25 clinical items incorporated five dimensions: EA, PA, SA, $\mathrm{EN}$, and PN. Sum score of five subscales were CTQ total score ranging from 25 to 125 , which indicated the severity of trauma exposure during childhood. Chinese version of CTQ-SF has good reliability and validity among Chinese undergraduates and depressive samples (22).

\section{The Pittsburgh Sleep Quality Index Questionnaire}

Pittsburgh Sleep Quality Index questionnaire, a self-rated questionnaire, was used to assess sleep quality and disturbances over 1 month (23). This scale consisted of 19 individual items which generate seven component scores: subjective sleep quality, sleep latency, sleep duration, sleep efficiency, sleep disturbances, use of sedative-hypnotic drugs, and daytime dysfunction. The sum of the scores for these seven components ranges from 0 to 21 , with a score $\leq 5,6-10,11-15$, and 16-21 indicating very good, fairly good, fairly poor, and very poor sleep quality, respectively.

\section{Impact of Event Scale-Revised}

Impact of Event Scale-Revised (IES-R), a self-administered questionnaire, was used to assess psychological impact of the COVID-19 epidemic on all participants. The IES-R included 22 items and consisted of three components: avoidance, intrusion, and hyperarousal. The total IES-R score was the sum score of the three components, with scores 0-23, 24-32, 33-36, and $>37$ indicating normal, mild, moderate, and severe psychological impact, respectively (24). The Chinese version of IES-R has been well-validated in the Chinese population, with high Cronbach's alpha coefficients for subscales [0.89 (intrusion), 0.85 (avoidance), and 0.83 (hyperarousal)] (25).

\section{Depression, Anxiety, and Stress Scale}

The Depression, Anxiety, and Stress Scale (DASS-21) was used to evaluate individual's mental health status in the last week. Depression, Anxiety, and Stress Scale included 21 items and could be divided into subscales of depressive, anxiety, and stress symptoms. The DASS-21 subscales were scored as follows: normal (0-9), mild (10-13), moderate (14-20), severe (21-27), and extremely severe (28+) for depression; normal (0-7), mild $(8,9)$, moderate (10-14), severe (15-19), and extremely severe $(20+)$ for anxiety; normal (0-14), mild (15-18), moderate (1925), severe (26-33), and extremely severe (34+) for stress (26). The Cronbach's alpha for Chinese version of DASS-21 was 0.95 for total scale, demonstrating a good internal consistency in the assessment of mental health in Chinese population (27).

\section{Statistical Analysis}

Pearson correlation analysis was conducted to calculate correlation coefficients between the total scale and five subscales of CTQ, perceived psychological impact of COVID-19 pandemic, and adverse mental health symptoms. Then, we applied a hierarchical linear regression to estimate the role of CTQ toward DASS-21, IES-R, and PSQI global sleep quality by sequentially adding predictors into two blocks within each model. The variables were added into models via the following steps: Step 1: input the demographic characteristics of age, sex, BMI, and education level; Step 2: add CTQ. Finally, a series mediation analysis was carried out to examine the mediated effects of perceived impact of the COVID-19 pandemic and PSQI global sleep quality in the relationship between childhood trauma and mental health status. The series mediation analysis was conducted by Process 3.5 for SPSS version 24.0 (model 6). The significance levels of direct, indirect, and mediated effects among the four factors [i.e., childhood trauma (X), the psychological impact of events (M1), PSQI global sleep quality (M2), and mental health parameters (Y)] were determined as two-tailed $p$-values $<0.05$, such figure being considered statistically significant in all other tests of this study. All continuous variables were standardized and then included in regression and mediation analyses performed on SPSS 24.0.

\section{RESULTS}

\section{Demographic Information, Childhood Trauma, COVID-19 Related Psychological Impact, Sleep Quality, and Mental Health Status}

After excluding invalid and incomplete questionnaires, 817 $(86.18 \%)$ of 948 participants were enrolled in this study. The mean age $( \pm$ SD) was $27.77( \pm 8.68)$ years old. The average education level and income were junior college and Low-medium income level, respectively. The mean scores of CTQ total scale and subscales of EA, PA, SA, EN, and PN were 37.03, 6.92, $6.12,5.55,9.77$, and 8.66, respectively. The mean scores of DASS-21 total scale and subscales of anxiety, depression, and stress were $14.56,4.15,4.96$, and 5.38, respectively. The mean scores of IES-R total scale and subscales of avoidance, intrusion, and hyperarousal were 9.04, 3.23, 3.40, and 2.40, respectively. The mean score of PSQI total score was 4.27. Of all subjects, there were $19.51,20.73$, and $9.76 \%$ individuals reporting mild to extremely severe anxiety, depressive, and stress symptoms, respectively. There were 25 (3.06\%), 38 (4.45\%), 10 (1.22\%), and $6(0.73 \%)$ subjects who were diagnosed by psychiatrists as depression, anxiety, bipolar disorder, and schizophrenia, respectively (Table $\mathbf{1}$ ).

\section{Correlations Among Childhood Trauma, COVID-19 Related Psychological Impact, Sleep Quality, and Mental Health Status}

Correlations of total scale scores of CTQ, PSQI sleep quality, DASS-21, and IES-R and subscales of those are displayed in Table 2. After Bonferroni correction, all the variables were 
TABLE 1 | Descriptive statistics, $N=817$.

\begin{tabular}{|c|c|c|c|c|}
\hline & Mean & SD & Min & Max \\
\hline Age & 27.77 & 8.68 & 16 & 59 \\
\hline Gender (M/F) & $431 / 386$ & & & \\
\hline $\mathrm{BMl}$ & 22.22 & 3.46 & 14.43 & 37.30 \\
\hline EDU level & 6.27 & 1.52 & 1 & 9 \\
\hline Income level & 2.44 & 0.85 & 1 & 5 \\
\hline \multicolumn{5}{|c|}{ Childhood Trauma Questionnaire (CTQ) } \\
\hline Mean CTQ score & 37.03 & 11.15 & 25 & 97 \\
\hline EA & 6.92 & 2.89 & 5 & 25 \\
\hline PA & 6.12 & 2.55 & 5 & 25 \\
\hline SA & 5.55 & 1.82 & 5 & 25 \\
\hline EN & 9.77 & 4.67 & 5 & 24 \\
\hline PN & 8.66 & 3.42 & 5 & 21 \\
\hline \multicolumn{5}{|c|}{ Depression, Anxiety, Stress and Stress Scale-21 (DASS-21) } \\
\hline Mean DASS-21 total score & 14.56 & 20.78 & 0 & 126 \\
\hline DASS-21 (anxiety) & 4.15 & 6.47 & 0 & 42 \\
\hline No $(0-7)$ & $657(80.42)$ & & & \\
\hline Mild $(8,9)$ & $35(4.28)$ & & & \\
\hline Moderate (10-14) & $76(9.30)$ & & & \\
\hline Severe (15-19) & $18(2.20)$ & & & \\
\hline Extremely severe $(20+)$ & $31(3.79)$ & & & \\
\hline Mean DASS-21 depression score & 4.96 & 7.61 & 0 & 42 \\
\hline No $(0-9)$ & $647(79.19)$ & & & \\
\hline Mild (10-13) & $66(8.08)$ & & & \\
\hline Moderate (14-20) & $67(8.20)$ & & & \\
\hline Severe $(21-27)$ & $14(1.71)$ & & & \\
\hline Extremely severe $(28+)$ & $23(2.82)$ & & & \\
\hline Mean DASS-21 stress score & 5.38 & 7.26 & 0 & 42 \\
\hline No $(0-14)$ & $737(90.21)$ & & & \\
\hline Mild (15-18) & $27(3.30)$ & & & \\
\hline Moderate (19-25) & $30(3.67)$ & & & \\
\hline Severe (26-33) & $16(1.96)$ & & & \\
\hline Extremely severe $(34+)$ & $7(0.86)$ & & & \\
\hline \multicolumn{5}{|c|}{ Impact of event scale-revised (IES-R) } \\
\hline Mean IES-R score & 9.04 & 10.49 & 0 & 88 \\
\hline Mean IES-R avoidance score & 3.23 & 4.19 & 0 & 32 \\
\hline Mean IES-R intrusion score & 3.40 & 4.13 & 0 & 32 \\
\hline Mean IES-R hyperarousal score & 2.40 & 3.39 & 0 & 24 \\
\hline PSQI global sleep quality score & 4.27 & 2.95 & 0 & 17 \\
\hline \multicolumn{5}{|c|}{ Self-reported diagnosed mental disorders by psychiatrists } \\
\hline Depression, $n(\%)$ & $25(3.06)$ & & & \\
\hline Anxiety, $n(\%)$ & $38(4.65)$ & & & \\
\hline Bipolar disorder, $n$ (\%) & $10(1.22)$ & & & \\
\hline Schizophrenia, $n$ (\%) & $6(0.73)$ & & & \\
\hline
\end{tabular}

BMI, body mass index; CTQ, childhood trauma questionnaires; DASS-21, depression, anxiety, stress and stress scale-21; EA, emotional abuse; EDU, education; EN, emotional neglect; IES-R, impact of event scale-revised; PA, physical abuse; PN, physical neglect; PSQI, Pittsburgh sleep quality index; SA, sexual abuse.

significantly correlated $(p<0.05)$ except for SA with DASS21 total scale, stress and depression subscales, IES-R total scale, avoidance, intrusion, and hyperarousal subscales, and PSQI total scale $(p>0.05)$; except for EN with IES-R avoidance subscale
( $p>0.05)$; and except for PN with IES-R avoidance subscale $(p>0.05)$. The total score of childhood trauma was positively and significantly associated with the perceived psychological impact of the pandemic, sleep quality, as well as DASS-21 anxiety, depression, and stress scores $(p<0.001)$. The PSQI total scale score was positively and significantly associated with the psychological impact of the pandemic, DASS-21 total score, and subscales of anxiety, depression, and stress scores $(p<$ $0.001)$. Moreover, the IES-R total scale score was positively and significantly associated with DASS-21 total scores and all subscale scores $(p<0.001)$.

\section{The Hierarchical Linear Regression Analysis of Psychological Impact Related to COVID-19, Mental Status, and Sleep Quality}

Table 3 shows the results of hierarchical linear regression analysis between childhood trauma and self-reported health status in all respondents, with adjustment to age, gender, BMI, and education level. In the hierarchical linear regression analysis, socioeconomic status, such as income level and educational level of parent(s), was incorporated into the model, but such model was not significant, thus excluding the socioeconomic factors from covariates in step 2. The final regression model (model 2) explained 17.3, 8.3, and 9.5\% of the total variance in DASS-21, IES-R, and PSQI total scale scores, respectively.

Total score of childhood trauma was a significant predictor for COVID-19 related psychological impact, mental status, and PSQI global sleep quality $(p<0.001)$.

\section{Series Mediation Effects of COVID-19 Related Psychological Impact and Sleep Quality Between Childhood Trauma and Mental Health Status}

Figure 1 shows the series mediating effect of psychological impact related to COVID-19 pandemic and PSQI global sleep quality in the association between childhood trauma and selfreported mental health status. All the paths in this model were significant $(p<0.001)$. First, the direct effect of childhood trauma on mental health status was explored, and the results showed that the model fitted well with $R^{2}=0.173, F=33.840$, and $p<0.001$. Specifically, childhood trauma can directly and positively predict mental health status $(\beta=0.362, p<$ 0.001 ). Second, considering childhood trauma as a independent variable, mental health status as the dependent variable, and psychological impact related to COVID-19 pandemic and PSQI global sleep quality as mediating variables, model (childhood trauma $\rightarrow$ psychological impact $\rightarrow$ sleep quality $\rightarrow$ mental health, see Figure 1) was thereby established. The mediation model exhibited acceptable goodness of fit $\left(R^{2}=\right.$ $0.504, F=116.845, p<0.001)$. This study further conducted the bootstrapping method for 5,000 times to test the significance of the mediating effect. The mediating effect was significant if the $95 \%$ confidence interval did not include 0 . The mediating effects of psychological impact and sleep quality on the 
TABLE 2 | Bivariate Pearson correlations with Bonferroni correction between main variables of interest.

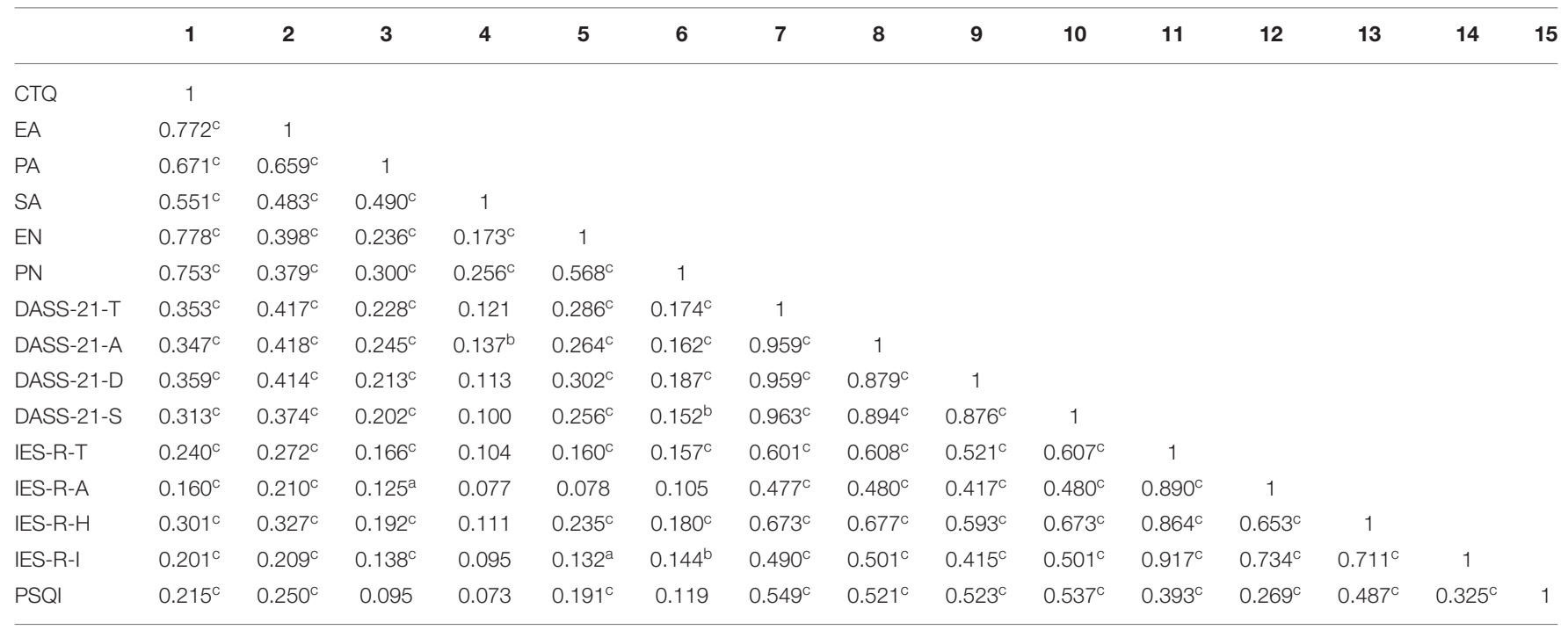

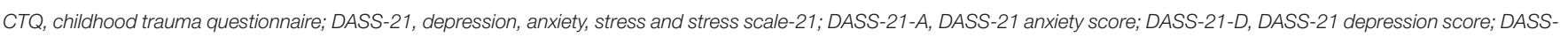

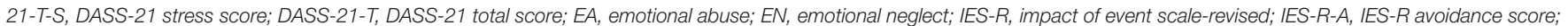

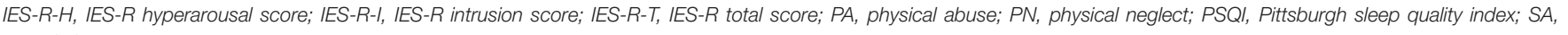
sexual abuse.

${ }^{a} p<0.05 ;{ }^{b} p<0.01 ;{ }^{c} p<0.001$.

TABLE 3 | Hierarchical regressions between CTQ total scale scores and DASS-21, IES-R, and PSQI global sleep quality scores.

\begin{tabular}{|c|c|c|c|c|c|c|c|c|c|}
\hline & \multicolumn{3}{|c|}{ DASS-21 } & \multicolumn{3}{|c|}{ IES-R } & \multicolumn{3}{|c|}{ PSQI global sleep quality } \\
\hline & $\beta$ & $S E$ & $P$ & $\beta$ & $S E$ & $P$ & $\beta$ & $S E$ & $\boldsymbol{P}$ \\
\hline Step 1 & \multicolumn{3}{|c|}{$\begin{array}{c}R^{2}=0.044, F=8.030, p= \\
0.000\end{array}$} & \multicolumn{3}{|c|}{$\begin{array}{c}R^{2}=0.021, F=4.039, p= \\
0.003\end{array}$} & \multicolumn{3}{|c|}{$\begin{array}{c}R^{2}=0.040, F=8.743, p= \\
0.000\end{array}$} \\
\hline Age & -0.085 & 0.035 & 0.014 & -0.029 & 0.041 & 0.471 & 0.009 & 0.037 & 0.810 \\
\hline Sex & 0.220 & 0.087 & 0.000 & 0.151 & 0.082 & 0.000 & 0.106 & 0.079 & 0.007 \\
\hline BMI & 0.075 & 0.043 & 0.086 & 0.113 & 0.041 & 0.006 & -0.014 & 0.039 & 0.727 \\
\hline Education & 0.026 & 0.024 & 0.480 & -0.009 & 0.024 & 0.812 & 0.150 & 0.023 & 0.000 \\
\hline Step 2 & \multicolumn{3}{|c|}{$\begin{aligned} R^{2}= & 0.173, \Delta R^{2}=0.129, F= \\
& 126.092, p=0.000\end{aligned}$} & \multicolumn{3}{|c|}{$\begin{array}{c}R^{2}=0.083, \Delta R^{2}=0.062, F= \\
54.771, p=0.000\end{array}$} & \multicolumn{3}{|c|}{$\begin{array}{c}R^{2}=0.095, \Delta R^{2}=0.055, F= \\
48.733, p=0.000\end{array}$} \\
\hline Age & -0.083 & 0.032 & 0.009 & -0.028 & 0.039 & 0.475 & 0.010 & 0.035 & 0.776 \\
\hline Sex & 0.189 & 0.080 & 0.000 & 0.129 & 0.078 & 0.001 & 0.086 & 0.076 & 0.024 \\
\hline BMI & 0.076 & 0.042 & 0.073 & 0.114 & 0.040 & 0.004 & -0.013 & 0.039 & 0.738 \\
\hline Education & 0.064 & 0.022 & 0.058 & 0.018 & 0.023 & 0.603 & 0.174 & 0.022 & 0.000 \\
\hline CTQ & 0.362 & 0.046 & 0.000 & 0.251 & 0.045 & 0.000 & 0.236 & 0.038 & 0.000 \\
\hline
\end{tabular}

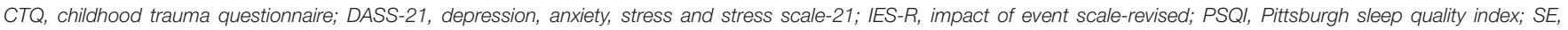
standard error.

association between childhood trauma and mental health status were significant (95\% confidence intervals were 0.095 [0.059, $0.136]$ and 0.051 [0.028, 0.078], respectively), and the series mediating effect was also significant 0.031 [0.020, 0.045]. The total mediation effect and the direct effect between childhood trauma and mental health were $0.178[0.128,0.228]$ and 0.184 $[0.133,0.235]$, respectively (Tables 4,5 ). Overall, the association between childhood trauma and mental health status was partly mediated by psychological impact and sleep quality (proportion explained $49.17 \%$ ).

\section{DISCUSSION}

To our knowledge, this is the first study using series mediation model to examine whether the association between childhood trauma and mental health status could be partly explained by COVID-19 pandemic related psychological impact and sleep quality. There were two findings worth highlighting. First, childhood trauma was a risk factor for COVID19 related psychological distress (avoidance, intrusion, and hyperarousal), sleep quality, and mental health status 


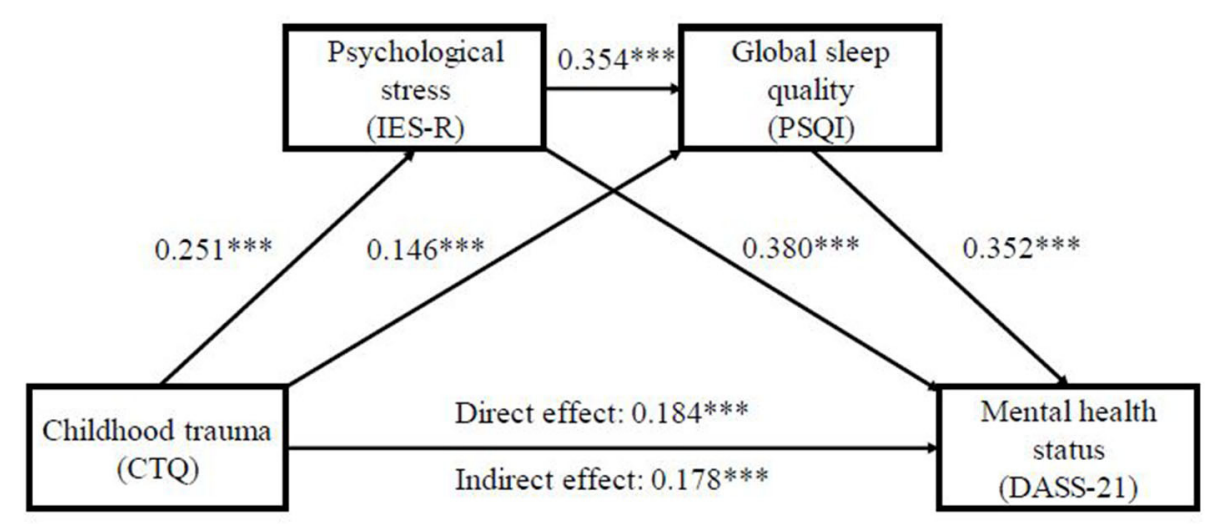

FIGURE 1 | Series mediation effects of psychological stress and sleep quality between childhood trauma and mental health status. CTQ, childhood trauma questionnaire; DASS-21, depression, anxiety, stress and stress scale-21; IES-R, impact of event scale-revised; PSQI, Pittsburgh sleep quality index. ${ }^{\star \star \star} p<0.001$.

TABLE 4 | Results of mediation analysis.

\begin{tabular}{|c|c|c|c|c|c|c|c|c|c|}
\hline & \multicolumn{3}{|c|}{ Fit index } & \multirow[b]{2}{*}{$B$} & \multirow[b]{2}{*}{$S E$} & \multirow[b]{2}{*}{$T$} & \multirow[b]{2}{*}{$p$} & \multicolumn{2}{|c|}{$\% 95 \mathrm{Cl}$} \\
\hline & $\boldsymbol{R}$ & $R^{2}$ & $\boldsymbol{F}$ & & & & & LLCI & ULCI \\
\hline \multicolumn{10}{|c|}{ Dependent variable: IES-R } \\
\hline CTQ & 0.289 & 0.083 & 146.88 & 0.251 & 0.034 & 7.400 & 0.000 & 0.185 & 0.318 \\
\hline Age & & & & -0.028 & 0.036 & -0.775 & 0.439 & -0.099 & 0.043 \\
\hline Sex & & & & 0.260 & 0.076 & 3.44 & 0.000 & 0.111 & 0.408 \\
\hline $\mathrm{BMl}$ & & & & 0.114 & 0.038 & 3.02 & 0.003 & 0.040 & 0.188 \\
\hline Education & & & & 0.012 & 0.023 & 0.521 & 0.602 & -0.033 & 0.056 \\
\hline Constant & & & & -0.456 & 0.171 & 2.665 & 0.008 & -0.792 & -0.120 \\
\hline \multicolumn{10}{|c|}{ Dependent variable: PSQI global sleep quality } \\
\hline CTQ & 0.458 & 0.210 & 35.697 & 0.162 & 0.033 & 4.494 & 0.000 & 0.082 & 0.210 \\
\hline IES-R & & & & 0.354 & 0.033 & 10.846 & 0.000 & 0.290 & 0.418 \\
\hline Age & & & & 0.020 & 0.034 & 0.593 & 0.553 & -0.046 & 0.086 \\
\hline Sex & & & & 0.080 & 0.071 & 1.128 & 0.260 & -0.059 & 0.218 \\
\hline BMI & & & & -0.053 & 0.035 & 1.514 & 0.131 & -0.122 & 0.016 \\
\hline Education & & & & 0.110 & 0.021 & 5.253 & 0.000 & 0.069 & 0.152 \\
\hline Constant & & & & -0.811 & 0.159 & 5.090 & 0.000 & -1.123 & -0.498 \\
\hline \multicolumn{10}{|c|}{ Dependent variable: DASS-21 } \\
\hline CTQ & 0.710 & 0.504 & 116.845 & 0.184 & 0.026 & 7.029 & 0.000 & 0.133 & 0.235 \\
\hline IES-R & & & & 0.380 & 0.028 & 13.692 & 0.000 & 0.325 & 0.434 \\
\hline PSQI & & & & 0.352 & 0.028 & 12.576 & 0.000 & 0.297 & 0.407 \\
\hline Age & & & & -0.078 & 0.027 & 2.921 & 0.004 & -0.131 & -0.026 \\
\hline Sex & & & & 0.221 & 0.056 & 3.945 & 0.000 & 0.111 & 0.331 \\
\hline BMl & & & & 0.037 & 0.028 & 1.328 & 0.185 & -0.018 & 0.092 \\
\hline Education & & & & -0.003 & 0.017 & -0.154 & 0.878 & -0.036 & 0.031 \\
\hline Constant & & & & -0.307 & 0.129 & 2.386 & 0.017 & -0.560 & -0.054 \\
\hline
\end{tabular}

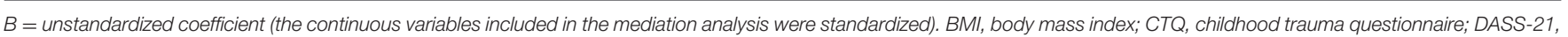
depression, anxiety, stress and stress scale-21; IES-R, impact of event scale-revised; PSQI, Pittsburgh sleep quality index; SE, standard error.

(depressive, anxiety, and stress symptoms). Moreover, after the effect of psychological impact related to COVID-19 pandemic was incorporated into the model, the impact of childhood trauma on mental health status was partly mediated by psychological distress for COVID-19 and global sleep quality.
The links between childhood trauma and poor sleep, depression, and anxiety were well-documented. One study found that severer childhood trauma was associated with poorer sleep health, including sleep quality, sleep efficiency, sleep duration, and daytime sleepiness (28). On the other hand, some cross-sectional studies found that childhood trauma was not only 
TABLE 5 | Results of the series mediating effects after bootstrapping test.

\begin{tabular}{lcccc}
\hline & \multicolumn{4}{c}{ Indirect Effect } \\
\cline { 2 - 5 } & $\beta$ & Se & LLCI & ULCI \\
\hline $\mathrm{CTQ} \rightarrow \mathrm{IES}-\mathrm{B} \rightarrow$ DASS-21 & & & & \\
$\mathrm{CTQ} \rightarrow \mathrm{PSQI} \rightarrow$ DASS-21 & 0.095 & 0.020 & 0.059 & 0.136 \\
$\mathrm{CTQ} \rightarrow \mathrm{IES}-\mathrm{P} \rightarrow$ PSQI $\rightarrow$ DASS-21 & 0.051 & 0.013 & 0.028 & 0.078 \\
& 0.031 & 0.006 & 0.020 & 0.045 \\
Total mediation effects & & & & \\
& 0.178 & 0.026 & 0.128 & 0.228 \\
\hline
\end{tabular}

CTQ, childhood trauma questionnaire; DASS-21, depression, anxiety, stress and stress scale-21; IES-R, impact of event scale-revised; PSQI, Pittsburgh sleep quality index; SE: standard error.

associated with depression and anxiety symptoms in the clinical samples and general population but also related to the onset and recurrence of depressive and anxiety disorders (29-31). Previous studies reported that dysfunction of HPA axis, cognitive emotion dysregulation, epigenetic regulation of the stress response, and abnormal change of brain structural and functional plasticity may create a barren climate for the development of mental health $(16,29,32,33)$.

This study highlighted that childhood trauma was associated with COVID-19 related psychological distress. A few studies strongly supported that severer childhood trauma prior to the COVID-19 pandemic predicted greater risks of occurring psychological symptoms $(17-19,34,35)$. One prospective study identified that childhood adverse experiences increased the risk of both psychological distress (ORs $=2.00-2.66)$ and probable acute stress reaction (ORs $=2.23-3.10)$ (5). Research evidence suggested that childhood trauma was associated with increased vulnerability to the stressful effect of the COVID-19 outbreak. Several potential mechanisms could interpret the association between the two. First, stress-vulnerability model assumes that childhood trauma, as chronic stress in the early life, could lower one's threshold of tolerance to acute stress, such as the outbreak of COVID-19 (16). Second, systemic inflammation may be an important mediator between childhood maltreatment and mental health in adulthood. Adults with exposure to childhood maltreatment exhibited a stronger inflammatory response under a standardized psychosocial stressor (36). Third, childhood trauma may change neuroendocrine responses to stress and increase vulnerability to acute events. One study found that limbic-medial temporal lobe regions, including amygdala and hippocampus, were sensitized in individual's exposure to life trauma. These regions could accommodate stress regulation/regulate stress and HPA axis function, and increase risk for negative stress-related mental health (37).

The results of the mediating effect test reveal that childhood trauma can affect adults' mental health through multiple mediating effects exerted by psychological impact of COVID-19 pandemic and sleep quality. Psychological impact of COVID19 pandemic played a mediating role in the association between childhood trauma and mental health. To put it in another way, childhood trauma can predict mental health not only in a direct way but also in an indirect way through COVID-19 related psychological impact and sleep quality. It was suggested by a previous study that insomnia symptoms partly mediate the relationship between childhood trauma and the severity of depressive and anxiety symptoms in patients with psychosis (20). This is also similar to the conclusion drew by John-Henderson that psychological stress mediated the association between childhood trauma and sleep quality (18). Childhood trauma is mainly related to the adaptive dimension of stress. This shows that people who experience severer childhood trauma are more likely to suffer a high degree of psychological impact. This result indicates that early intervention in children's exposure to trauma may reduce the occurrence of event-related trauma symptoms (38). Therefore, childhood trauma can lead to greater COVID-19 related psychological impact and, thus, poorer mental health status. The results of this study confirmed the series mediating roles of COVID-19 related psychological impact and PSQI global sleep quality in the association between childhood trauma and mental health.

There are several limitations in this study that should be noted. First, this study was based on self-reported questionnaires, which could lead to self-awareness and reporting bias. Second, due to the cross-sectional design in this study, the causal relationships between childhood trauma and mental health may be weakened. Third, the COVID-19 related changes of people's life (e.g., family conflicts and unemployment) were not included in our study. Therefore, the impact of these confounding factors on sleep quality and mental health could not be controlled. Fourth, the associations between childhood trauma and mental health are also affected by other mental disorders, which are not controlled in our analysis. Longitudinal design needs to specify the direction of the relationships between childhood trauma and mental health or experimentally observe childhood trauma to explore the resultant changes in mental health in the following research.

\section{CONCLUSION}

This study found that childhood trauma was positively associated with COVID-19 related psychological impact (avoidance, intrusion, and hyperarousal), anxiety, depression, and stress symptoms. In addition, the results of the series mediation analysis indicated an underlying mechanism of the association between childhood trauma and mental health: severer childhood trauma was able to predict poorer mental health outcomes via COVID19 pandemic related psychological impact and sleep quality. Early interventions should be implemented to raise public awareness of adverse consequences of childhood trauma, whether emotional or physical, and poor mental health via family and social strategies. Extra attention should be paid to individuals who are experiencing or have experienced trauma or bully in the past. In addition, psychiatrists should also take patients' history of childhood trauma into consideration when making treatment decisions/in clinical decision-making. 


\section{DATA AVAILABILITY STATEMENT}

The original contributions presented in the study are included in the article/supplementary material, further inquiries can be directed to the corresponding author/s.

\section{ETHICS STATEMENT}

The studies involving human participants were reviewed and approved by Sichuan University. Written informed consent for participation was not provided by the participants' legal guardians/next of kin because: Written informed consent is difficult to obtain because we collected data via Wechat online survey. Because the questionnaire was anonymous, we assumed that participants consented to participate in our study by returning the questionnaire.

\section{REFERENCES}

1. Castellini G, Cassioli E, Rossi E, Innocenti M, Gironi V, Sanfilippo G, et al. The impact of COVID-19 epidemic on eating disorders: a longitudinal observation of pre versus post psychopathological features in a sample of patients with eating disorders and a group of healthy controls. Int J Eat Disord. (2020) 53:1855-62. doi: 10.1002/eat.23368

2. Huang C, Huang L, Wang Y, Li X, Ren L, Gu X, et al. 6-month consequences of COVID-19 in patients discharged from hospital: a cohort study Lancet. (2021) 397:220-32. doi: 10.1016/S0140-6736(20)32656-8

3. Kang L, Ma S, Chen M, Yang J, Wang Y, Li R, et al. Impact on mental health and perceptions of psychological care among medical and nursing staff in Wuhan during the 2019 novel coronavirus disease outbreak: a cross-sectional study. Brain Behav Immun. (2020) 87:11-7. doi: 10.1016/j.bbi.2020.03.028

4. Song X, Fu W, Liu X, Luo Z, Wang R, Zhou N, et al. Mental health status of medical staff in emergency departments during the coronavirus disease 2019 epidemic in China. Brain Behav Immun. (2020) 88:605. doi: 10.1016/j.bbi.2020.06.002

5. Li Y, Wang Y, Jiang J, Valdimarsdóttir UA, Fall K, Fang F, et al. Psychological distress among health professional students during the COVID19 outbreak. Psychol Med. (2021) 51:1952-4. doi: 10.1017/S00332917200 01555

6. Deng J, Zhou F, Hou W, Silver Z, Wong CY, Chang O, et al. The prevalence of depression, anxiety, and sleep disturbances in COVID-19 patients: a meta-analysis. Ann N Y Acad Sci. (2021) 1486:90-111. doi: 10.1111/ny as. 14506

7. Olff M, Primasari I, Qing Y, Coimbra B, Hovnanyan A, Grace E, et al. Mental health responses to COVID-19 around the world. Eur J Psychotraumatol. (2021) 12:1929754. doi: 10.1080/20008198.2021.1929754

8. McKay M, Cannon M, Chambers D, Conroy R, Coughlan H, Dodd P, et al. Childhood trauma and adult mental disorder: a systematic review and metaanalysis of longitudinal cohort studies. Acta Psychiatr Scand. (2021) 143:189205. doi: 10.1111/acps.13268

9. Kajeepeta S, Gelaye B, Jackson CL, Williams MA. Adverse childhood experiences are associated with adult sleep disorders: a systematic review. Sleep Med. (2015) 16:320-30. doi: 10.1016/j.sleep.2014.12.013

10. Matheson SL, Shepherd AM, Pinchbeck RM, Laurens KR, Carr VJ. Childhood adversity in schizophrenia: a systematic meta-analysis. Psychol Med. (2013) 43:225-38. doi: 10.1017/S0033291712000785

11. Woolley CS, Gould E, McEwen BS. Exposure to excess glucocorticoids alters dendritic morphology of adult hippocampal pyramidal neurons. Brain Res. (1990) 531:225-31. doi: 10.1016/0006-8993(90)90778-A

\section{AUTHOR CONTRIBUTIONS}

MX, YT, and LZ contributed to the writing of this article and the statistical analysis. QW and TL led the whole study, including carrying out this study, and putting forward the study. MD, YW, YH, YL, and LX contributed to the data collection and statistical analysis. All authors contributed to editing the manuscript and have approved the final manuscript.

\section{FUNDING}

This study was funded by the National Natural Science Foundation of China (grant no. 81771446 and 82171499).

\section{ACKNOWLEDGMENTS}

We would like to thank all participants for their contributions and members of the research team for their help in data collection.

12. Gomes FV, Zhu X, Grace AA. The pathophysiological impact of stress on the dopamine system is dependent on the state of the critical period of vulnerability. Mol Psychiatry. (2020) 25:3278-91. doi: 10.1038/s41380-019-0527-9

13. Hill MN, Eiland L, Lee TTY, Hillard CJ, McEwen BS. Early life stress alters the developmental trajectory of corticolimbic endocannabinoid signaling in male rats. Neuropharmacology. (2019) 146:154-62. doi: 10.1016/j.neuropharm.2018.11.036

14. Niwa M, Jaaro-Peled H, Tankou S, Seshadri S, Hikida T, Matsumoto Y, et al. Adolescent stress-induced epigenetic control of dopaminergic neurons via glucocorticoids. Science. (2013) 339:335-9. doi: 10.1126/science.1226931

15. Mayo D, Corey S, Kelly LH, Yohannes S, Youngquist AL, Stuart BK, et al. The role of trauma and stressful life events among individuals at clinical high risk for psychosis: a review. Front Psychiatry. (2017) 8:55. doi: 10.3389/fpsyt.2017.00055

16. Walker EF, Diforio D. Schizophrenia: a neural diathesis-stress model. Psychol Rev. (1997) 104:667-85. doi: 10.1037/0033-295X.104.4.667

17. John-Henderson NA, Ginty AT. Historical trauma and social support as predictors of psychological stress responses in American Indian adults during the COVID-19 pandemic. J Psychosom Res. (2020) 139:110263. doi: 10.1016/j.jpsychores.2020.110263

18. John-Henderson NA. Childhood trauma as a predictor of changes in sleep quality in American Indian adults during the COVID-19 pandemic. Sleep Health. (2020) 6:718-22. doi: 10.1016/j.sleh.2020.09.001

19. Siegel A, Lahav Y. Emotion regulation and distress during the COVID19 pandemic: the role of childhood abuse. I Interpers Viol. (2021) 2021:8862605211021968. doi: 10.1177/08862605211021968

20. Laskemoen JF, Aas M, Vaskinn A, Berg AO, Lunding SH, Barrett EA, et al. Sleep disturbance mediates the link between childhood trauma and clinical outcome in severe mental disorders. Psychol Med. (2020) 2020:110. doi: 10.1017/S0033291720000914

21. Bernstein DP, Stein JA, Newcomb MD, Walker E, Pogge D, Ahluvalia $\mathrm{T}$, et al. Development and validation of a brief screening version of the childhood trauma questionnaire. Child Abuse Negl. (2003) 27:16990. doi: 10.1016/S0145-2134(02)00541-0

22. He J, Zhong X, Gao Y, Xiong G, Yao S. Psychometric properties of the Chinese version of the Childhood Trauma Questionnaire-Short Form (CTQSF) among undergraduates and depressive patients. Child Abuse Negl. (2019) 91:102-8. doi: 10.1016/j.chiabu.2019.03.009

23. Buysse DJ, Reynolds CF III, Monk TH, Berman SR, Kupfer DJ. The Pittsburgh sleep quality index: a new instrument for psychiatric practice and research. Psychiatry Res. (1989) 28:193-213. doi: 10.1016/0165-1781(89)90047-4 
24. Creamer M, Bell R, Failla S. Psychometric properties of the impact of event scale-revised. Behav Res Ther. (2003) 41:148996. doi: 10.1016/j.brat.2003.07.010

25. Wu KK, Chan KS. The development of the Chinese version of impact of event scale-revised (CIES-R). Soc Psychiatry Psychiatr Epidemiol. (2003) 38:94-8. doi: 10.1007/s00127-003-0611-x

26. Hao F, Tan W, Jiang L, Zhang L, Zhao X, Zou Y, et al. Do psychiatric patients experience more psychiatric symptoms during COVID-19 pandemic and lockdown? A case-control study with service and research implications for immunopsychiatry. Brain Behav Immun. (2020) 87:100-6. doi: 10.1016/j.bbi.2020.04.069

27. Jiang LC, Yan YJ, Jin ZS, Hu ML, Wang L, Song Y, et al. The depression anxiety stress scale-21 in chinese hospital workers: reliability, latent structure, and measurement invariance across genders. Front Psychol. (2020) 11:247. doi: 10.3389/fpsyg.2020.00247

28. Brindle RC, Cribbet MR, Samuelsson LB, Gao C, Frank E, Krafty RT, et al. The relationship between childhood trauma and poor sleep health in adulthood. Psychosom Med. (2018) 80:200-7. doi: 10.1097/PSY.00000000000 00542

29. Huh HJ, Kim KH, Lee HK, Chae JH. The relationship between childhood trauma and the severity of adulthood depression and anxiety symptoms in a clinical sample: the mediating role of cognitive emotion regulation strategies. J Affect Disord. (2017) 213:44-50. doi: 10.1016/j.jad.2017. 02.009

30. McKinley CE, Boel-Studt S, Renner LM, Figley CR. Risk and protective factors for symptoms of depression and anxiety among American Indians: understanding the roles of resilience and trauma. Psychol Trauma. (2021) 13:16-25. doi: 10.1037/tra0000950

31. Hovens JG, Giltay EJ, Spinhoven P, van Hemert AM, Penninx BW. Impact of childhood life events and childhood trauma on the onset and recurrence of depressive and anxiety disorders. J Clin Psychiatry. (2015) 76:9318. doi: 10.4088/JCP.14m09135

32. Houtepen LC, Vinkers CH, Carrillo-Roa T, Hiemstra M, van Lier PA, Meeus W, et al. Genome-wide DNA methylation levels and altered cortisol stress reactivity following childhood trauma in humans. Nat Commun. (2016) 7:10967. doi: 10.1038/ncomms 10967

33. McEwen BS, Nasca C, Gray JD. Stress effects on neuronal structure: hippocampus, amygdala, and prefrontal cortex. Neuropsychopharmacology. (2016) 41:3-23. doi: 10.1038/npp.2015.171
34. Li X, Lv Q, Tang W, Deng W, Zhao L, Meng Y, et al. Psychological stresses among Chinese university students during the COVID-19 epidemic: the effect of early life adversity on emotional distress. J Affect Disord. (2021) 282:33-8. doi: 10.1016/j.jad.2020.12.126

35. Kim AW, Nyengerai T, Mendenhall E. Evaluating the mental health impacts of the COVID-19 pandemic: perceived risk of COVID-19 infection and childhood trauma predict adult depressive symptoms in urban South Africa. Psychol Med. (2020) 2020:1-13. doi: 10.1017/S0033291720003414

36. Carpenter LL, Gawuga CE, Tyrka AR, Lee JK, Anderson GM, Price LH. Association between plasma IL-6 response to acute stress and earlylife adversity in healthy adults. Neuropsychopharmacology. (2010) 35:261723. doi: $10.1038 /$ npp. 2010.159

37. Seo D, Rabinowitz AG, Douglas RJ, Sinha R. Limbic response to stress linking life trauma and hypothalamus-pituitaryadrenal axis function. Psychoneuroendocrinology. (2019) 99:3846. doi: 10.1016/j.psyneuen.2018.08.023

38. Kelleher I, Keeley H, Corcoran P, Ramsay H, Wasserman C, Carli $\mathrm{V}$, et al. Childhood trauma and psychosis in a prospective cohort study: cause, effect, and directionality. Am J Psychiatry. (2013) 170:73441. doi: 10.1176/appi.ajp.2012.12091169

Conflict of Interest: The authors declare that the research was conducted in the absence of any commercial or financial relationships that could be construed as a potential conflict of interest.

Publisher's Note: All claims expressed in this article are solely those of the authors and do not necessarily represent those of their affiliated organizations, or those of the publisher, the editors and the reviewers. Any product that may be evaluated in this article, or claim that may be made by its manufacturer, is not guaranteed or endorsed by the publisher.

Copyright (c) 2021 Xie, Tang, Zhu, Dai, Wu, Huang, Liu, Xiao, Li and Wang. This is an open-access article distributed under the terms of the Creative Commons Attribution License (CC BY). The use, distribution or reproduction in other forums is permitted, provided the original author(s) and the copyright owner(s) are credited and that the original publication in this journal is cited, in accordance with accepted academic practice. No use, distribution or reproduction is permitted which does not comply with these terms. 